\title{
More Threatening and More Diagnostic: How Moral Comparisons Differ from Social Comparisons Supplemental Material
}

\section{Overview}

In this supplemental material, we an additional experiment. Experiment S1 tests humanity as a boundary condition for the diagnosticity of moral comparisons.

\section{Experiment S1: When does Diagnosticity Operate in Moral Comparisons?}

Experiments 5a and 5b demonstrate that traditional boundaries of diagnosticity are reduced in morality. However, we do not want to claim that diagnosticity plays no role in moral comparisons at all. Instead, factors that determine diagnosticity for morality are unique to morality, the most important one being that morality is a panhuman standard, a standard that applies to all humans - but only humans. Therefore, in Experiment S1, we test whether comparisons are still relevant when they are made with non-humans - in this case, aliens.

\section{Method}

Participants and design. 583 MTurker (355 female, 226 male, 2 other; $M_{\text {age }}=33 ; 76 \%$ White, 8\% African American, 6\% Hispanic, 10\% other) participated for a compensation of $\$ 0.25$. Sample size was set to 560 a priori, based on the effect size of Experiment S1b, a power of $80 \%$ (calculated with GPower, Faul et al., 2007), and the assumption that there would be no effect in the non-diagnostic comparison conditions (Simonsohn, 2014). Again, participants were assigned to one cell of a 2(Threat: low vs. high; between) x 2(Comparison Standard: upward vs. downward, within) x 2(Standard Diagnosticity: diagnostic vs. non-diagnostic, between) mixed design.

Materials. We used moderate comparisons for both conditions, but in the diagnostic condition, these stories were from MTurkers, while in the non-diagnostic condition the stories were from aliens. The aliens were introduced as the Hraxksl, sentient gas clouds who live millions of light years away. The descriptions of the stories were the same, except that in the non-diagnostic condition, some words were replaced by Hraxksl words. For example, the MTurker story "Stole the computer from Dan" was paralleled by the Hraxksl story "Stole the Xraks from Klasifg" (see Materials on https://osf.io/fd6at/?view_only=9298bd746f97465f 80583d2643ceb594).

Measures. We used the previous measures of interest and choice of stories, as in Experiment 4b. At the end, participants filled out demographics.

\section{Results}

A 2(Threat: low vs. high; between) x 2(Comparison Standard: upward vs. downward, within) x 2(Standard Diagnosticity: diagnostic vs. nondiagnostic, between) mixed ANOVA on interest in stories showed the expected two-way interaction of threat with comparison standard, $F(1,579)$ $=20.22, p<.001, \eta_{p}^{2}=.034, \mathrm{CI}_{90}[.014, .061]$. More importantly, supporting our predictions, this interaction was qualified by the predicted threeway interaction of threat, comparison standard and standard diagnosticity, $F(1,579)=26.39, p<$ $.001, \eta_{p}^{2}=.044, \mathrm{CI}_{90}[.020, .074]$. Separate analy- 
ses conducted in the diagnostic and non-diagnostic conditions showed that we replicated the threat $\mathrm{x}$ comparison standard interaction in the diagnostic (MTurker) condition, $F(1,292)=32.79, p<.001$, $\eta_{p}^{2}=.101, \mathrm{CI}_{90}[.052, .157]$, but not in the nondiagnostic (aliens) condition, $F(1,287)=0.36, p$ $=.549, \eta_{p}^{2}=.001, \mathrm{CI}_{90}[<.001, .017]$, supporting our predictions that all comparisons are threatening as long as they are human.

In the diagnostic condition, simple effects analyses replicated that participants in the low-threat condition preferred moral stories over immoral stories, $F(1,292)=50.16, p<.001, \eta_{p}^{2}=.147, \mathrm{CI}_{90}[.089$, $.208]$. In contrast, participants who were threatened seemed to prefer downward comparisons more, as they did not prefer either moral or immoral stories, $F(1,292)=0.83, p=.363, \eta_{p}^{2}=.003, \mathrm{CI}_{90}$ $[<.001, .022]$. However, in the non-diagnostic condition, participants always preferred reading the moral stories over reading the immoral stories, $F(1$, $287)=8.52, p=.004, \eta_{p}^{2}=.029, \mathrm{CI}_{90}[.006, .068]$ for the low-threat condition, $F(1,287)=13.27, p$ $<.001, \eta_{p}^{2}=.044, \mathrm{CI}_{90}[.013, .089]$ for the highthreat condition.

Looking at choice, in line with our predictions, the effects in the diagnostic and non-diagnostic conditions differed significantly, as shown by a significant interaction between threat and diagnosticity, $b=$ $0.91, p=.008, \mathrm{CI}_{95}[0.254,1.532]$. In the diagnostic condition, we replicated the previous effect: Participants in the low-threat condition choose moral stories more often than immoral ones (33\% immoral stories), but in the high-threat condition, participants more often chose immoral stories (56\% immoral stories), $\chi^{2}(1)=15.46, p<.001, \phi=.23$, $\mathrm{CI}_{95}[.12, .34]$. Supporting our predictions, in the non-diagnostic condition, this effect was not significant, $\chi^{2}(1)=0.02, p=.896, \phi=.01, \mathrm{CI}_{95}[-.12$, .11]. Both when threatened and when not, participants in the non-diagnostic condition choose moral stories more often than immoral ones (both conditions $63 \%$ ).

\section{Discussion}

Experiment S1 demonstrates that not all comparisons in morality are relevant, just human comparisons. Consistent with the notion of morality being a panhuman standard, we found that participants did not avoid upward moral comparisons of an alien race. Moral standards are often considered absolute, so absolute that they apply to everyone, regardless of time and space, and therefore moral comparisons with extreme and distant comparisons become relevant. However, moral standards clearly apply only to humans; therefore, comparisons with standards outside the group of humans are not threatening, and not avoided.

In line with this idea that morality acts as a panhuman standard, it would be interesting to test whether the same moral behavior, but performed by non-human agents, is perceived as threatening. Non-human agents, such as animals, computers, or robots, do not fall under the pan-human standard and so their "moral" behavior should not be threatening. However, people often anthropomorphize non-human agents (Waytz et al., 2010), assigning human-like attributes to them and making them part of the human group. Therefore, it would be interesting to test whether the threat of a nonhuman standard increases with the ease by which the non-human agent can be anthropomorphized. For example, imagine a robot that is programmed to spend money, and "decides" to spend thousands of dollars on a donation. It is possible that people experience different levels of threat when comparing to this robot, depending on their tendency to anthropomorphize the robot, and therefore judge it under the panhuman moral standard. People who anthropomorphize the robot strongly would feel threatened by its moral behavior, while people who do not would only see a robot following its programming. This has obvious implications for selfdriving cars and other recent inventions that have made such moral questions relevant. For example, smart speakers like Siri or Alexa, or even cars that are designed to be anthromorphized, might threaten people's moral identity and lead to people avoiding them. 